\title{
A rare case presentation for congenital pseudarthrosis of the collar bone
}

\begin{abstract}
Congenital pseudarthrosis of the clavicle (CPC) is a rare diagnosis of undetermined etiologies with a limited number of cases were reported. Classically, children patients presenting asymptomatic with a diagnosis that can be confirmed by X-rays with the absence of a central zone portion of the clavicle. Although clinical presentations are variable, which ranges from being asymptomatic to discomfort or sometimes restriction in shoulder range of motion, thus, some cases reported manifestations of thoracic outlet syndromes. Therefore, Management of such cases is individually tailored from conservative to surgical intervention when indicated in overt symptomatic patients or those with significant deformity. Here, we present a case of right-sided CPC in a 13-year-old male patient detected as he concerned about its appearance, which diagnosed and managed non-surgically with further follow- up in our hospital.
\end{abstract}

Volume I2 Issue I - 2020

\section{Fahad AI Hulaibi, Zainab Al Eid, Khalid Shoubair, Ahmed Siddique}

Department of orthopedic, National Guard Hospital, Saudi Arabia

Correspondence: Fahad Al Hulaibi, Department of orthopedic, King Abdulaziz of National Guard Hospital,Al Ahsa, Saudi Arabia,Tel 0096650 1876716, Email dr.fahad.hu@gmail.com

Received: February 07, 2020 | Published: February 17, 2020

Keywords: clavicle, congenital, pseudarthrosis

Abbreviations: CPC, congenital pseudarthrosis of the clavicle; $\mathrm{CCD}$, craniocleidodysostosis

\section{Background \& Summary}

Congenital pseudarthrosis of the clavicle (CPC) is a rare congenital malformation that was first described by FitzWilliams in $1910 .{ }^{1}$ The second case reported by Saint-Pierre reported in a female patient diagnosed after birth for having right side CPC in $1930 .^{2}$ The incidence of these cases not well reported, which attributed to its infrequent presentation. In the case series, Owen's reported 33 cases and Cadhilac 25 cases. ${ }^{3,4}$ This condition is considered a diagnostic challenge as it results in children of a difficult delivery, which sometimes diagnosed as a fracture secondary to birth trauma. At this moment, we present the first case diagnosed with congenital pseudarthrosis of the clavicle in our institution.

\section{Case presentation}

A 13-year-old male presented to the clinic accompanied by his father, he was following up after the left distal radius buckle fracture. During the general examination, there is a painless mass on the right middle third of the clavicle. Thus, he did not undergo any form of treatment until the presenting age. The patient was born by spontaneous delivery, and there was no history of recent trauma or birth difficulties. He is also medically and surgically free.

On examination, a deformity on the middle third of the right clavicle was noted, which is localized and non-tender, cosmetically unappealing mass (Figure 1). No further masses or deformities identified anywhere else. No skin lesions or areas of hyperpigmentation were appreciated. There was a normal function of the right shoulder with no restriction of power or movement (Figure 1). The sensation was intact to the right hand, and grip strength was symmetric. The remainder of the examination was unremarkable.
Plain radiographs of the right clavicle (Figure 2) at this time reported as showing a 'hypertrophic non-union of the clavicle of which the lateral component formed the bony lump in the right supraclavicular fossa'. At this stage, he was asymptomatic. Therefore, the decision was made to manage him conservatively, and he is arranged for further follow-up subsequently.
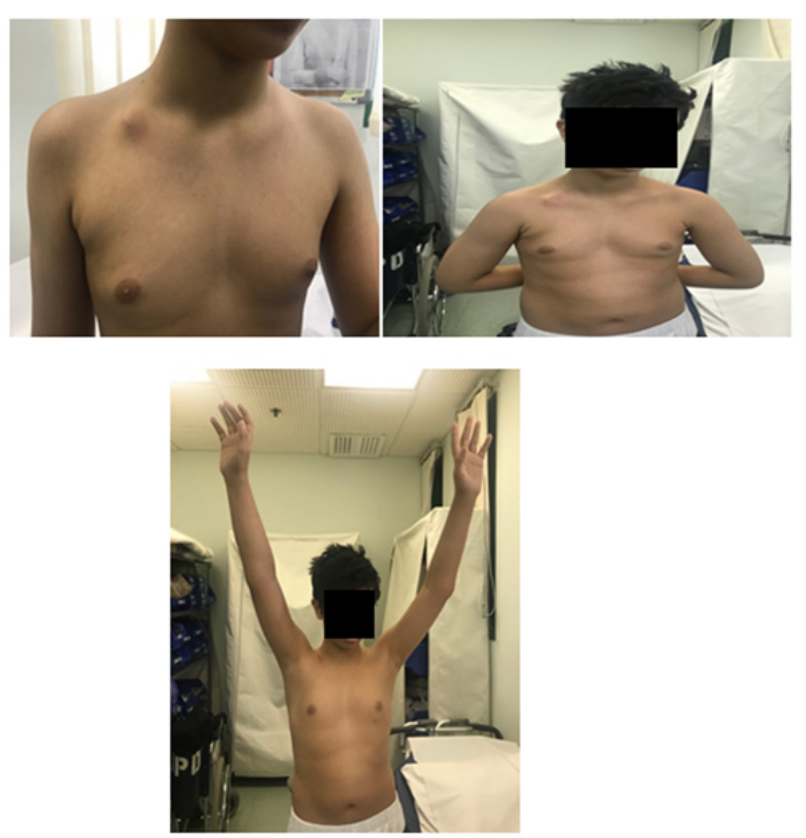

Figures I Clinical photo shows the right side clavicle mass with full range of motion of right shoulder. 

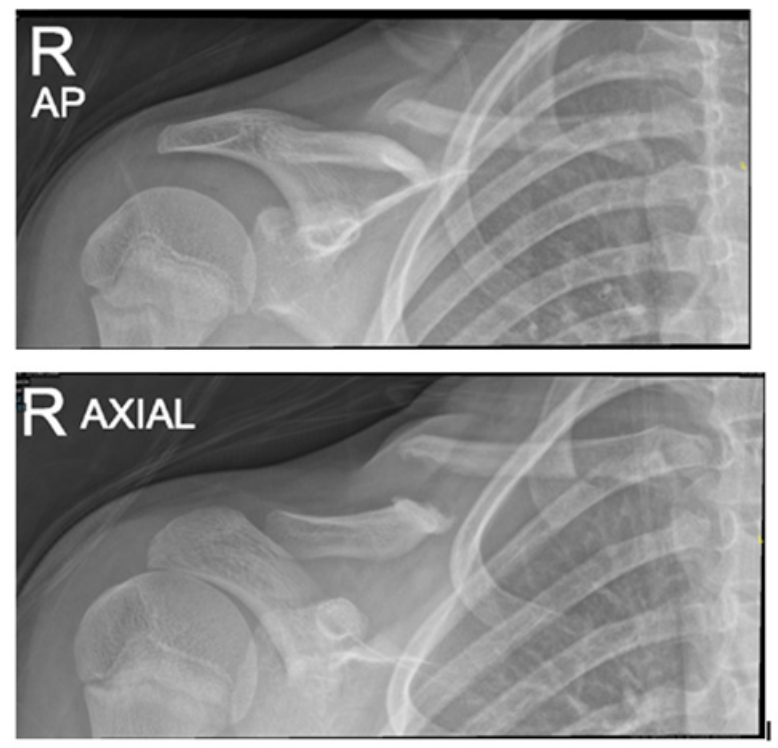

Figures 2 X-ray showing right clavicle pseudarthrosis with demonstration of non-healed mid third clavicular fracture. The vertical distance between the fractured fragments about I.2 with overlapping segment between the fractured fragment about $1.4 \mathrm{~cm}$.

\section{Discussion}

The first case of CPC was documented in 1910, the second case reported in $1930 .{ }^{1,2}$ CPC is described as a clinical condition that is distinct from craniocleidodysostosis (CCD), obstetric delivery difficulties birth trauma resulting in secondary fractures and neurofibromatosis. ${ }^{5,6}$ It was documented that CPC has an association with cervical ribs with roughly $15 \%$ of diagnosed cases. ${ }^{7}$ However, our case showed no evidence of a cervical rib. Thus, it is a rare entity but classically documented as being a right-sided condition. ${ }^{6}$ With left-sided cases usually associated with dextrocardia. ${ }^{7}$ To the best of our knowledge, no cases published yet in literature from Saudi Arabia.

However, one case series reported that men and women are equally affected. Although this is exceedingly rare, there are distinguished cases reported the existence of bilateral pseudarthrosis. ${ }^{8}$ These cases associated with a strong familial predisposition, suggesting that genetics may play a role in the development of CPC. ${ }^{9-13}$

The etiology is still not well understood and undetermined risk factors to identify the pathogenesis, but the final consensus has not been established. ${ }^{10}$ Familial cases, CPC in combination with other abnormalities such as cleidocranial dysplasia or neurofibromatosis, and the possible involvement of the RUNX2 (CBFA1) gene mutation has been found in the literature. ${ }^{12,13}$ Cases presented classically asymptomatic, but there are cases progressed with complications that might occur, such as thoracic outlet obstruction, although it is a rare sequela. ${ }^{14}$ Proper history taking with consistent examinations and findings of plain radiographs are fair enough to diagnose this condition. Importantly, to differentiate this condition from others, more severe syndromes such as Cleidocranial dysostosis is an autosomal dominant condition that affects the $6 \mathrm{p} 21$ gene, encoding the RunX2 transcription factor. Furthermore, hallmarks of the syndrome include multiple skeletal disorders, and Hypoplasia of the clavicles is a hallmark of the syndrome.
The treatment for pseudarthrosis of the clavicle is individually tailored from being conservative with observation and follow up in asymptomatic cases to surgical interventions whenever as needed. In many cases, the natural history is benign, and no urgent intervention is necessary. However, the cosmetic issue may indicate surgical interventions. ${ }^{15,16}$ We decided to manage our patient conservatively with follow up as there are cases documented progression from benign condition to development of thoracic outlet syndrome with late presentations. ${ }^{14}$ In such cases, more immediate surgical intervention may be warranted. Although no clear decision regarding the time of proceeding with surgical intervention. A published Study had explored three cases surgically, which concluded the existence of clinical differences between earlier versus late operation. Therefore, recommendations tend to perform an excision of the pseudarthrosis, filling the void using an autologous bone graft, and using an anatomical bridging plate. ${ }^{17}$

\section{Conclusion}

CPC is a rare anomaly that classically occurs in the right clavicle of male children and presents as a non-tender and cosmetically unappealing mass. Treatment ranges according to the patients' presentations from observation in those cases with a mild unnoticeable deformity and surgical resection with fixation in those with severe deformity and complications.

\section{Acknowledgments}

None.

\section{Conflicts of interest}

The authors declare that there are no conflicts of interest.

\section{References}

1. FitzWilliams DC. Hereditary craniocleidodysostosis. Lancet. 1916;11:466.

2. Kite JH. Congenital pseudarthrosis of the clavicle. South Med J. 1968;61(7):703-710.

3. Owen R. Congenital pseudarthrosis of the clavicle. J Bone Joint Surg Br. 1970;52(4):644-652.

4. Cadilhac C, Fenoll B, Peretti A, et al. Congenital pseudarthrosis of the clavicle: 25 childhood cases. Rev Chir Orthop Reparatrice Appar Mot. 2000;86(6):575-580.

5. Al-Hadidy A, Haroun A, Al-Ryalat N, et al. Congenital pseudarthrosis associated with venous malformation. Skeletal Radiol. 2007;36:S15$\mathrm{S} 18$

6. Alldred AJ. Congenital pseudarthrosis of the clavicle. J Bone Joint Surg Br. 1963;45B:312-319.

7. Sakkers RJ, Tjin a Ton E, Bos CF. Left-sided congenital pseudarthrosis of the clavicula. J Pediatr Orthop. 1999;8(1):45-47.

8. Figueiredo MJPSS, Braga SR. Congenital pseudarthrosis of the clavicle: updating article. Rev Bras Ortop. 2012;47(1):21-26.

9. Toledo LC, MacEwen GD. Severe complication of surgical treatment of congenital pseudarthrosis of the clavicle. Clin Orthop. 1979;139:64-67.

10. Lloyd-Roberts GC, Apley AG, Owen R. Reflections upon the aetiology of congenital pseudarthrosis of the clavicle. With a note on cranio-cleido dysostosis. J Bone Joint Surg Br. 1975;57(1):24-29. 
11. Fairbank HA. Cranio-cleido-dysostosis. J Bone Joint Surg Br. 1949;31B(4):608-617.

12. O'Leary E, Elsayed S, Mukherjee A, et al. Familial pseudarthrosis of the clavicle: does it need treatment? Acta Orthop Belg. 2008;74(4):437-440.

13. Price BD, Price CT. Familial congenital pseudarthrosis of the clavicle: case report and literature review. Iowa Orthop J. 1996;16:153-156.

14. Watson HI, Hopper GP, Kovacs P. Congenital pseudarthrosis of the clavicle causing thoracic outlet syndrome. BMJ Case Rep. 2013.
15. Persiani P, Molayem I, Villani C, et al. Surgical treatment of congenital pseudarthrosis of the clavicle: a report on 17 cases. Acta Orthop Belg. 2008;74(2):161-166.

16. Sung TH, Man EM, Chan AT, et al. Congenital pseudarthrosis of the clavicle: a rare and challenging diagnosis. Hong Kong Med J. 2013;19(3):265-267.

17. Giwnewer U, Rubin G, Dohovni E, et al. Congenital pseudarthrosis of the clavicle: Should we reconsider timing and fixation method? J Orthop Surg (Hong Kong). 2018;26(3):1-7. 